\title{
FIBRE OPTIC SENSING AS INNOVATIVE TOOL FOR EVALUATING RAILWAY TRACK CONDITION?
}

\author{
I. Vidovic $^{1 *}$, M. Landgraf ${ }^{2}$ \\ ${ }^{1}$ Institute of Railway Engineering and Transport Economy, Graz University of Technology, Graz, Austria \\ ${ }^{2}$ Institute of Railway Engineering and Transport Economy, Graz University of Technology, Graz, Austria \\ * Corresponding author
}

\begin{abstract}
The condition and deterioration of a railway track over time has a major influence on its maintenance demands, service life and consequently its life cycle costs. Railway track condition is currently assessed on the basis of manual inspections, wayside equipment and measurements performed by a measurement car. This paper deals with combining the advantages of above assessment technologies, allowing continuous and permanent measurements for the entire network. On the one hand, we use Distributed Acoustic Sensing, a methodology relying on the effect of Rayleigh backscattering. This technology uses fibre optic cables, which are already installed in cable troughs alongside railway tracks and used for telecommunication or signalling. On the other hand, fractal analysis of vertical track geometry allows for a componentspecific condition evaluation, i.e. distinguishing the root cause of an irregularity in track geometry. Correlating both methodologies should show whether or not/to what and to what extent this innovative methodology of using fibre optic cables is applicable for assessing the component specific condition of a railway track. The proposed combination of both methodologies paves the way for real time condition assessment of railway track.
\end{abstract}

\section{Introduction}

Railway infrastructure managers face various challenges in the world today. One of the main challenges is the success of railway operations: An increase in traffic volume leads to both increasing wear of track components and shortened track closures for maintenance activities. The former results in higher maintenance and renewal demands while the latter is a consequence of higher track possession due to increased traffic volume. This means that fewer time slots for maintenance are available. However, these time slots are crucial for the necessary maintenance work in order to keep track quality at a required level. Since we do not wish to argue the case for decreasing rail traffic, we - in the railway infrastructure sector - must find a way to be more efficient in maintaining railway track. The evaluation and assessment of a railway track is a decisive task for infrastructure managers. Moreover, in times of budget cutbacks, it is becoming more important to implement a sustainable and condition based maintenance strategy based on life cycle cost considerations. The evaluation and assessment of a railway track are vital tasks providing essential information for any railway infrastructure manager. The knowledge about track condition as well as distinguishing the specific component responsible for the end of service life forms the base for any sustainable decision making (life cycle management). (Marschnig et al., 2006; Veit, 2006). Such a strategy can only be effectively implemented when frequent track measurements are carried out, since these are the key to data quality and quantity. For the assessment of a railway track infrastructure managers have access to several data sources, providing them with information on the track condition with varying degrees of data quality and quantity. Manual inspections; e.g. for turnouts and crossing noses; are carried out at intervals of several weeks for each turnout. This delivers a relatively large quantity of data for one asset but is also highly subjective and therefore not reproducible as while also time and staff resource costly. Besides manual inspections, a track-recording vehicle assesses the entire network a few times each year and delivers data of high quality and quantity (e.g. Figure 1). Wayside Train Monitoring Systems and the equipping of points of interests with sensors have become very popular over the past few years. This type of information delivers permanent measurements for each train passing but only for specific points within the network. Permanent measurements such as axle counters deliver information at any point in time but only at fixed positions. On the contrary, continuous measurements such as track-recording cars deliver information at one point in time but for a certain longitude.

Figure 1 Data source and availability

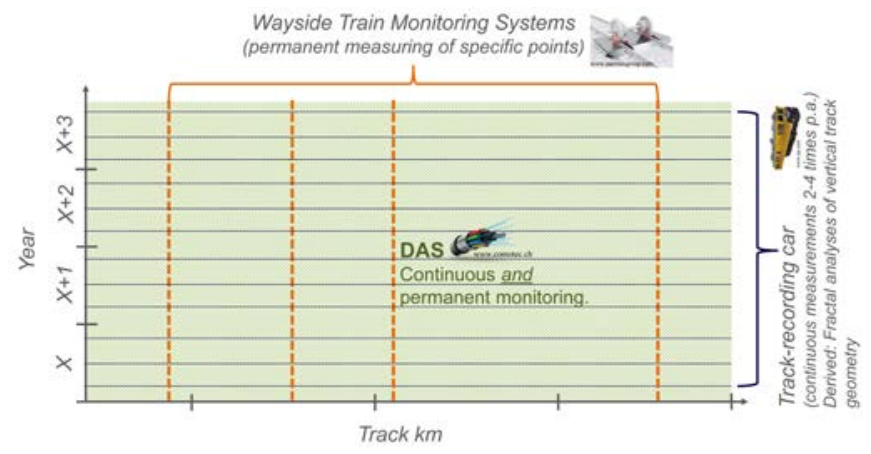


In this paper, we describe a methodology for combining both the advantages of a track-recording vehicle and those of sensors provided alongside railway tracks allowing continuous and permanent measurements for the whole network.

Distributed Acoustic Sensing relies on the effect of Rayleigh backscattering. It uses fibre optic cables (mainly used for telecommunication or signalling) which are already installed in cable troughs, and fractal analysis (Landgraf and Hansmann, 2018) of vertical track geometry. Fractal analysis of vertical track geometry enables the assessment of the component specific track condition based on longitudinal level, which is measured by any state-of-the-art trackrecording car. Thus, correlating the information from Distributed Acoustic Sensing with the evaluations based on fractal analysis of vertical track geometry should show whether or not/to and to what extend this innovative methodology of using fibre optic cables is applicable for assessing condition of railway track. This would be a breakthrough for track asset management as fibre optic cables could deliver continuous and permanent information on railway track status. While it cannot replace track-recording cars, since these deliver a variety of measurement signals, this methodology could allow constant monitoring between the measurement runs. Moreover, this methodology detects every passing train and thus can provide decisive additional information for the infrastructure manager such as track behaviour for different traffic loads or the starting and ending points of maintenance measures. This would be a major step towards predictive maintenance and would lead to higher availability on railway tracks and more efficient maintenance strategies.

\section{Fractal analysis of vertical track geometry}

This analysis methodology is based on the fact that vertical track geometry can be derived from a sum of many parts of harmonic irregularities with different wavelengths and their amplitudes. Commonly used indices for describing track condition such as mean values or standard deviations (European Committee for Standardization, 2014) focus on the amplitude of defects within the alignment, but neglect the wavelength, i.e. the characteristic of a failure. Already starting in 1984 (Frederich, 1984), an attempt was made to analyse the failure characteristics using power spectral density. By using a Fast Fourier Transformation this method allows the recorded measurement signal to be split into its periodic components in order to determine the corresponding amplitude for each wavelength range. Its network-wide and automatized implementation would be difficult to achieve, however, for two main reasons: (1) the complexity of this procedure makes it difficult to convert it into an automatized process, applying it in a sliding approach for a whole network especially would involve even more of an effort; (2) due to its level of detail several values arise from this approach describing each present wavelength range and its amplitudes. These various numbers of values are an obstacle to one of the main challenges in creating quality figures from measurement signals for railway applications: Keep the information as detailed as necessary, but also as simple to understand as possible. Fractal analysis of vertical track geometry realizes the aforementioned challenge by delivering expressive and informative quality figures in a comprehensible way.

\subsection{Methodology of fractal analyses of vertical track geometry}

The methodology of fractal analysis is not new. Mandelbrot (Mandelbrot, 1967) developed it in 1967 as an answer to an essentially fictional problem - without thinking of its possible benefits for the rail industry. His considerations centred on a historical question: the exact length of the coastline of Great Britain. As it was impossible to approximate the length of the karstic coastline of Great Britain with sufficient accuracy using Euclidean geometry, Mandelbrot tried to solve the problem using a polygonal chain. This is how he developed the Modified Divider Method (Mandelbrot and Blumen, 1989).

In 2002, Hyslip (Hyslip, 2002) realised the potential this method had for analysing track geometry and applied the calculation method to a corridor section of AMTRAK.

Figure 2 Polygonal chain and measuring signal

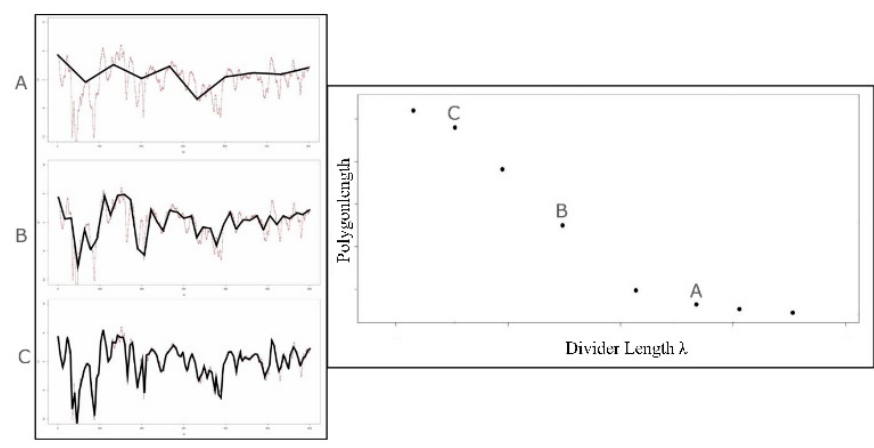

This new calculation method approximates the existing measuring signal using a polygonal chain composed of partial segments of uniform length. The length of the partial segments is reduced systematically. As a result, the polygonal chain corresponds more and more closely to the measuring signal (Figure 2).

These adjustments and the correlation between the length of the partial segments and the length of the resulting polygonal chain form a typical Richardson plot. Mandelbrot already used this methodology to calculate the so-called fractal dimension of a curve. The fractal dimension of a curve increases with its roughness. In a Richardson plot it is quantified by means of a tangent using the log-log comparison of the polygon length and the segment length.

Studies carried out by Graz University of Technology show how the Richardson plot can be used to describe with sufficient precision, three sectors using tangents (Landgraf and Hansmann, 2018). The calculation of the tangent slope in individual sections allows assessing the roughness of the signal and consequently the impact of different wave-length ranges on the complete signal (Figure 6). 
Figure 3 Divider length and polygonlength

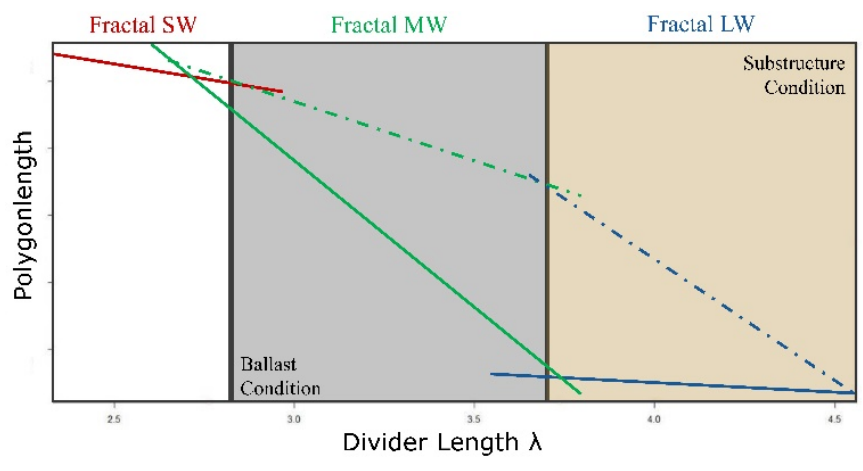

A high fractal value (i.e. a high slope in the correspondent range) indicates problems within ballast and / or substructure (Landgraf, 2018). In Figure 3, the dotted line represents a higher slope within the long-wave range, which implies that a substructure issue is apparent. The continuous line shows nearly no slope within the long-wave, but a high slope within the mid-wave sector, which is an indicator of problems concerning the ballast bed.

The wavelength ranges were chosen according to (European Committee for Standardization, 2014) as shown in Table 1:

Table 1 Chosen wavelength ranges

\begin{tabular}{lll}
\hline Wavelength range & From $[\mathrm{m}]$ & To $[\mathrm{m}]$ \\
\hline Short-wave range & 1 & 3 \\
Mid-wave range & 3 & 25 \\
Long-wave range & 25 & 70 \\
\hline
\end{tabular}

These wavelength ranges are valid for permissible speeds in Austria (up to $250 \mathrm{~km} / \mathrm{h}$ ). However, executing fractal analyses for high speed lines $(>300 \mathrm{~km} / \mathrm{h})$ may require the adding of a fourth sector within the calculation and handling wavelengths of more than $70 \mathrm{~m}$ separately. These parameters (three slopevalues) define every single cross-section using a defined influence length. Thus, net-wide analyses can be conducted by executing fractal analyses of vertical track geometry as part of a gliding algorithm for every track section over the network. Therefore, track section specific evaluations are possible as well as evaluations of segments, lines and networks.

\subsection{Application and validation}

This method could be analysed on a large scale for the first time in Austria. This was possible due to the Institute of Railway Engineering and Transport Economy of Graz University of Technology developing a data warehouse consisting of the main network in close cooperation with Austrian Federal Railways (ÖBB). This data warehouse consists of both asset information and measurement data recorded since 2002. Thus, fractal analyses could be applied to 4,000 track-kilometres with a time series of 14 years. Within the scope of these analyses it was possible to link evaluations from fractal analyses of vertical track geometry to net-wide asset data as well as data from Ground Penetrating Radar (GPR) for 1,400 track kilometres (Landgraf, 2018).

In 2016, the methodology of fractal analyses was also implemented within swissTAMP, which is the tool for track analyses and maintenance planning of Swiss Federal Railways (SBB). This allowed working with data containing another $4,500 \mathrm{~km}$ of track with time series since 2007. Thanks to the SBB geotechnical department it was possible to correlate fractal analyses to net-wide geotechnical surveys conducted within the last five years (Figure 4).

Figure 4 Correlation net-wide geotechnical surveys and fractal analysis
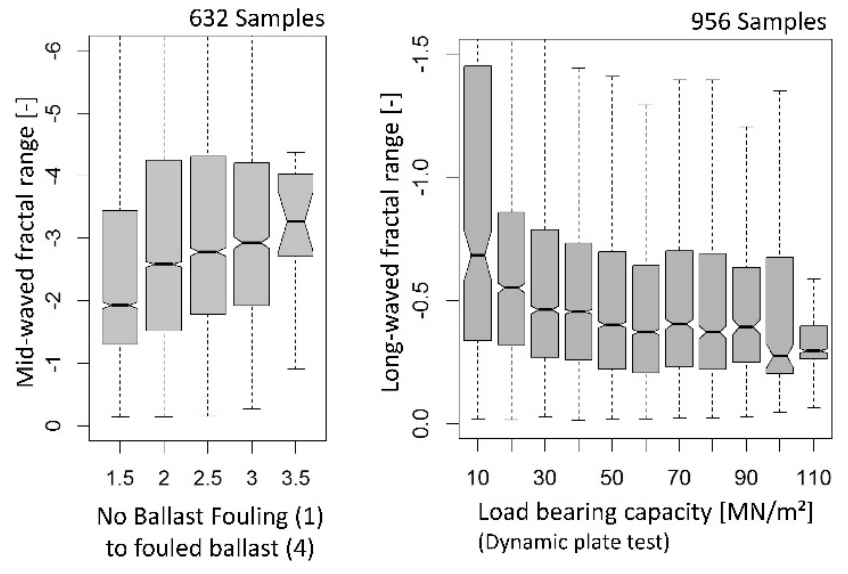

The left illustration of Figure 4 shows evaluations of 632 ballast samples which could be correlated to the mid-waved fractal value. Within these ballast samples the proportion of fines - i.e. degree of ballast fouling - was tested on every cross section. The correlation analysis shows that the higher the degree of ballast fouling, the higher the values for the midwaved range of fractal analyses. Thus, fractal analyses enable evaluation of the ballast condition.

Similar analyses could be conducted for the long-waved fractal value (Figure 4, right). This value - evaluating the substructure condition - was correlated with the load bearing capacity measured by dynamic plate tests. These correlation analyses prove that the higher the load bearing capacity - i.e. the better the in-situ condition of the substructure - the lower the values of the long-waved fractal range.

Consequently, it can be stated that fractal analyses are able to evaluate the condition of the substructure. These evaluations require only the vertical track geometry, a measurement signal which is already available to any railway infrastructure manager. 


\section{Distributed Acoustic Sensing (DAS)}

Distributed Acoustic Sensing is a special form of fibre optic cable sensing. It relies on the effect of Rayleigh backscattering, an elastic scattering process of light (Bao and Chen, 2012). The fibre optic cable itself represents the sensing element and it can be described as a listening device with thousands of "virtual" microphones situated along the monitored asset. The system is capable of detecting, locating and classifying any activity taking place nearby or on the monitored asset by using any conventional telecommunication single mode fibre optic cable. For railway applications the system can make use of the fibre optic cables already installed alongside railway tracks for telecommunication and signalling purposes. This eliminates the need of additional equipment on or alongside a track. In its beginnings Distributed Acoustic Sensing was commonly used for the monitoring of leakage and third party intrusions along oil and gas pipelines and recently also for border and perimeter monitoring (Owen et al., 2012; Vale and Rauscher, 2013; Hill, 2015). As this technology emerges within the railway business first algorithms have been developed for train tracking and wheel flat identification (Lienhart et al., 2016; Papp et al., 2016; Wiesmeyr et al., 2018). Since this field of application has only started recently, all of the algorithms mentioned are currently executed under fixed boundary conditions. On the one hand, this means the cable is always mounted equally distanced from track which results in a steady signal quality while within a railway network this distance varies. The distance between track and fibre optic cable highly affects the signal quality. Consequently, there is a high correlation between the position of the fibre optic cable and the signal. This fact will be explained in detail in section 2.2.1. On the other hand, tests under fixed boundary conditions allow for a minimization of perturbations which also the signal quality. However, recent tests have already shown a possible potential of this technology for condition based maintenance strategies of railway infrastructure (Rosenberger, 2018).

\subsection{Methodology of DAS}

The interrogation system which was part of this first in-situ application utilizes Coherent Optical Time Domain Reflectometry (C-OTDR) (Ip et al., 2008) to reconstruct the acoustic signal present along the monitored asset. One interrogation unit is capable of monitoring up to $40-50 \mathrm{~km}$ of connected optical fibre. As the acoustic signal is distributed on an equidistant basis at thousands of locations along the monitored asset, the system reconstructs the acoustic signals from the inherent Rayleigh backscatter with the result that a light pulse is transmitted through an optical fibre. Depending on the activities and events taking place down an optical fibre the system reconstitutes the acoustic signal from the observed backscattered light signal (Owen et al., 2012).

The described monitoring device is suitable for monitoring of extended assets as several interrogator units can be connected with one single fibre optic cable. A benefit of DAS is that a strong signal near the sensor has no impact either on receiving signals further along the sensing cable or on the signal quality. This means that the system is impervious to linear variation.

\subsection{Fields of application in railways}

DAS offers a vast area of applications, especially in railways. In the safety and security context it enables detection of environmental anomalies like landslides, rock falls or avalanches and also cable theft and third party intrusions (Schubert and Bauer, 2015; Pohl and Schubert, 2017).

3.2.1 Train tracking: In the specific case two interrogator units monitor a track of almost 41 kilometres whereby an installed optical fibre cable laid in a cable trough alongside the track is used. Figure 5 displays a waterfall diagram, which is in fact a real-time diagram of a graphic timetable, of the monitored track. The $\mathrm{x}$-axis represents track kilometres, whereat one track kilometre consists of 100 channels which means a $10 \mathrm{~m}$ spatial resolution and the $y$-axis represents the time. As shown, Figure 5 illustrates a 30-minute recording between 8:30-09:00 AM. Since it is an acoustic sensing system the colour scheme represents the level of acoustic intensity and whether it is a high or low acoustic event. A blue colour indicates a low-level acoustic intensity and red one a high-level acoustic intensity. Furthermore, the acoustic level of a passing train depends on the distance between the track on which the trains run and the cable trough where the fibre optic cable is laid. The closer the sensing element is positioned to the monitored track the higher the acoustic level of a passing train. This fact becomes evident on the first ten kilometres in Figure 5. In this section the cable trough is situated slightly further away from the track and this can be seen within the acoustic signal. For train tracking it is not only important to know the exact speed of a train but also the duration of stops at stations. Within the monitored section of 41 kilometres the trains in operation pass eleven stations at which, depending on the train type, they can stop for passengers. When a train stops the acoustic intensity of the signal decreases and this also becomes visible in the colour scheme. Additionally, it is possible to distinguish between train types whether if it is a freight train or a passenger train. Moreover, braking and acceleration processes can be analysed which is also shown in Figure 5.

Figure 5 30-minute waterfall diagram of $41 \mathrm{~km}$ track

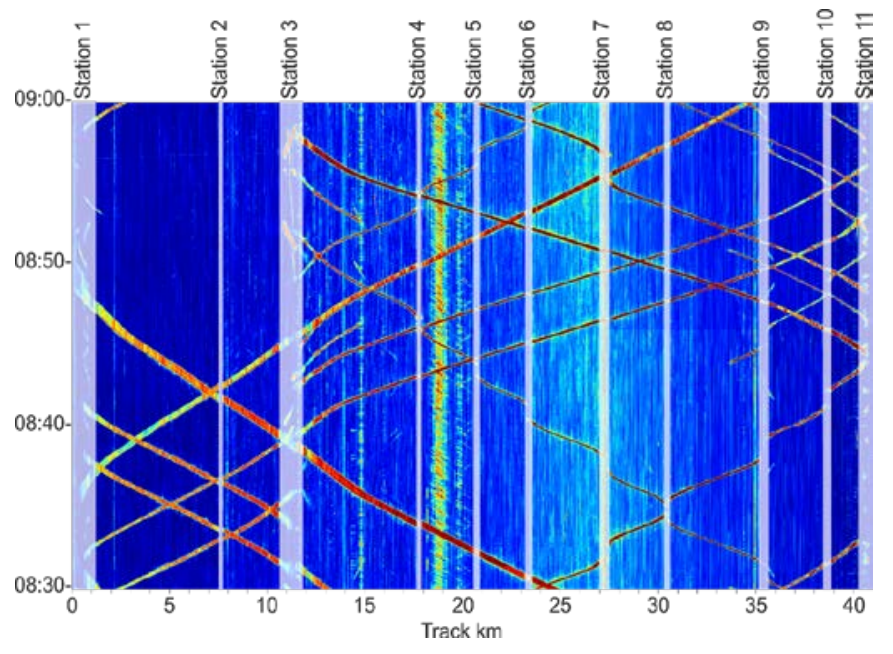


Using DAS for train tracking and detection is very well described in the literature (Lauer and Stein, 2015; Timofeev et al., 2015; Papp et al., 2016).

3.2.2 Railway infrastructure condition monitoring: While the acoustic signal of DAS for the purpose of train tracking has been thoroughly examined, its use for monitoring of railway infrastructure is a relatively new topic. The first step is thus to examine whether there is a difference in the raw acoustic signal in regards to railway track condition. Consequently, the hypothesis is that the same train passing a track section in good condition and a track section showing poor condition leads to different values within the raw signal. In detail, we assume for instance that a train passing a worn out crossing nose of a turnout generates a louder acoustic signal than when passing a crossing nose in proper condition or a track section with good track quality and no worn components (e.g. ballast breakdown, white spots, etc.). Additionally, according to this theory the signal pattern among those two assets should also differ when the same train passes these points.

The result of the described theory is depicted in Figure 6 where 14 seconds of the raw acoustic signal of a passing train are illustrated. The $y$-axis represents the intensity of the signal in arbitrary unit and the $\mathrm{x}$-axis the time. It can be stated that the acoustic intensity of the same train passing an open track section in proper condition is far below the acoustic intensity level of a train passing a worn crossing nose (e.g. Figure 6). However, the lower acoustic level of an open track section in good condition scatters much more than the acoustic signal of a train passing a worn crossing nose. This can be explained by the fact that a higher acoustic intensity leads to pure measurement signal. In contrast to that, a lower acoustic intensity shows a higher scattering since the vibration is not as dominant. Consequently, the significance of the scattering within the signal should be part of further research. For the time being, however, we are focusing on the actual level of the acoustic intensity.

Figure 6 Level of intensity based on raw data

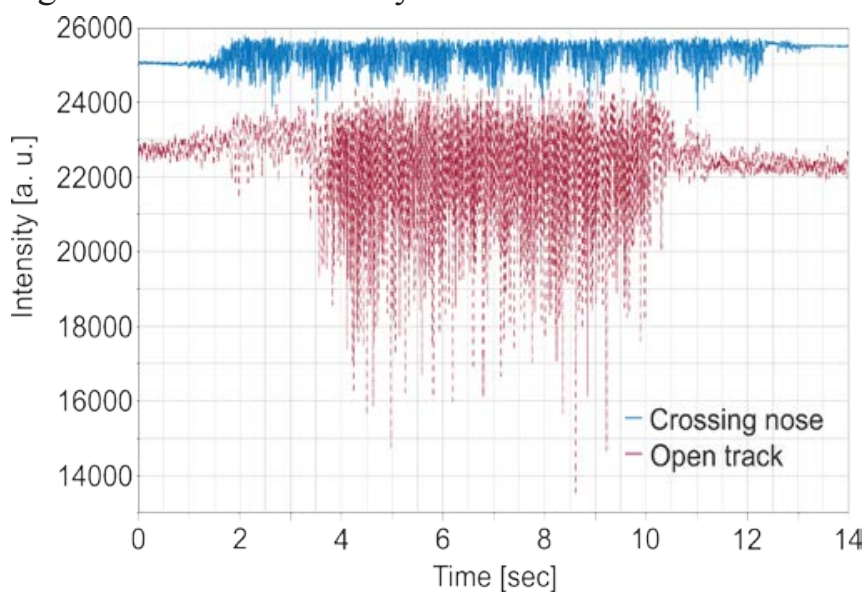

3.2.3 Maintenance work monitoring In many cases, infrastructure managers only have insufficient information on maintenance work carried out such as grinding or tamping. This relates to information such as section length including the exact starting and ending points of the maintenance work. This kind of information is sometimes either not properly recorded and stored or it is held exclusively by the maintenance companies.

However, monitoring of maintenance work is a very specific form of train traffic monitoring and therefore it is possible to utilize DAS for this purpose. As already shown for train detection (Figure 5) maintenance works can also be illustrated within a waterfall diagram. The acoustic level and therefore the operational intensity of a maintenance machine is significantly higher than the intensity of any train.

Figure 7 depicts detailed analyses of a monitored tamping action. One tamping process consists of 1-3 squeezing processes and the relocation of the tamping unit. Furthermore, the tasks ballast penetration, squeezing movement and lifting are part of one squeezing process. The aim was to examine whether it is possible to see individual squeezing processes per sleeper and thus their frequency within the acoustic signal. Additionally, the variation of a squeezing process per sleeper should be examined. Within the displayed 30 seconds, the system recorded three tamping processes. The number of the underlying squeezing processes as well as the squeezing time depends on two parameters: (1) the current and the desired track quality and (2) the experience of the maintenance staffmembers. As clearly visible for the first sleeper, one squeezing process was necessary to achieve a specific track quality (between seconds 0 to 10 ). Subsequently the tamping unit is relocated to the next sleeper. The second sleeper shows two squeezing processes with a variation in the squeezing time, whereby the second squeezing process took slightly longer than the first one.

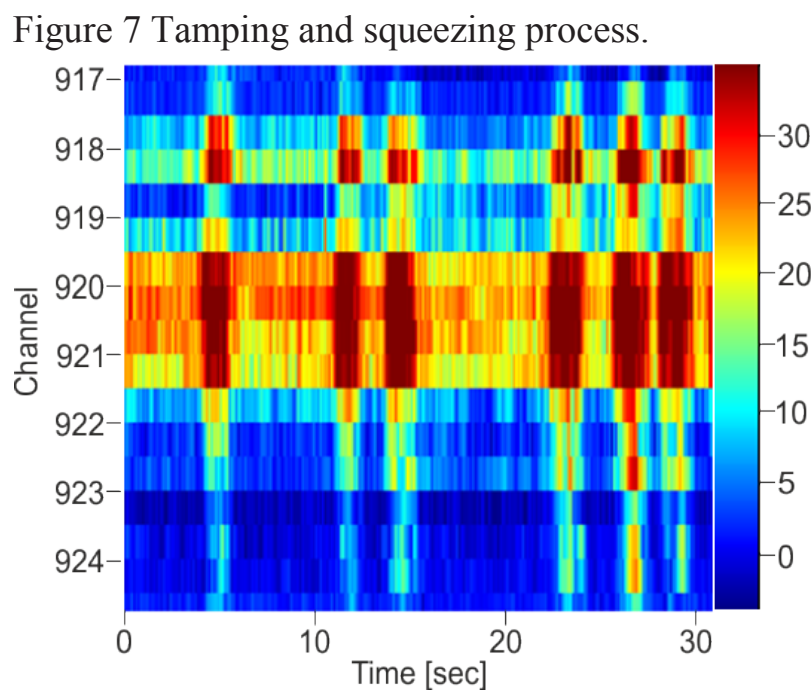
30 25 20 霁

Finally, for the third sleeper three squeezing processes with longer squeezing movements were necessary to attain a specific track quality.

Apart from train tracking or safety and security purposes, these current results demonstrate the potential of this technology for infrastructure managers in the context of railway track asset management. Thus, for the first time infrastructure managers 
have the opportunity to monitor executed maintenance works in detail while simultaneously being able to store and analyse the data. This allows for gathering information but also for linking it with other data sources already available in order to understand and predict the holistic and component specific behaviour of railway track. Linking the data obtained from DAS and track recording cars, such as fractal analyses of vertical track geometry, could well allow for continuous and permanent description and prediction of railway track condition.

\section{Results}

As previously outlined, a correlation of Distributed Acoustic Sensing and fractal analysis of vertical track geometry should show the potential of DAS for a component specific condition assessment of railway track. We analysed a track section in poor ballast condition for the validation of the fibre optic acoustic signal. Figure 8 shows white spots at kilometre 126.230 around the sleepers, which result from ballast that has been crushed as a result of overstress. Presumably, there is already a very large amount of fine-grained material (crushed ballast) in the ballast bed and the bottom edge of the sleeper may be worn out resulting in an inadequate load transfer.

Figure 8 White spot in ballast bed $\mathrm{km} 126.230$

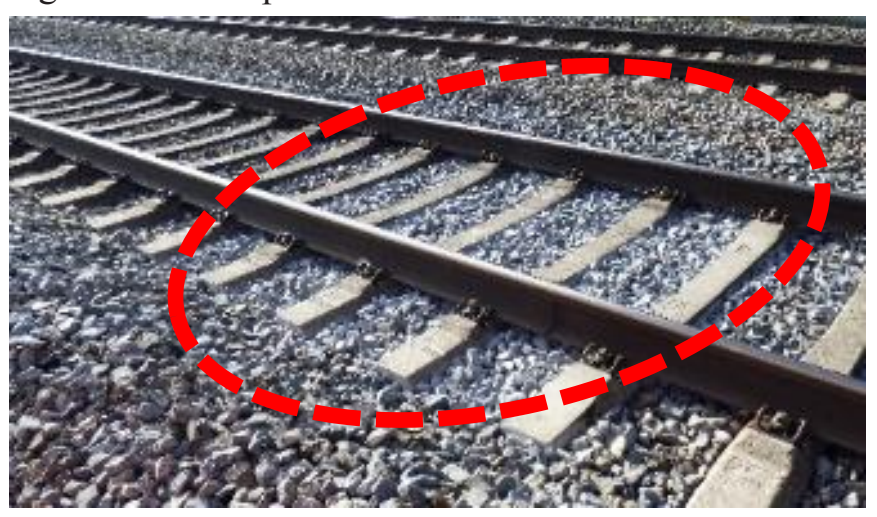

As stated in chapter three, the mid-waved fractal range is an indication of problems concerning the ballast bed. Figure 9 illustrates results of analyses of the mid-waved fractal dimension for a $500 \mathrm{~m}$ track section where the previously described failure occurs. Within a fractal analysis plot the yaxis represents the fractal value and the $\mathrm{x}$-axis the longitude in kilometres. The smaller the mid-waved fractal value, the poorer the ballast condition. Figure 9 clearly shows that the lowest values for mid-waved fractal analysis of vertical track geometry are situated around $\mathrm{km} \mathrm{126,230.} \mathrm{This} \mathrm{indicates} \mathrm{poor}$ ballast condition in this area and confirms the white spots shown in Figure 8. Landgraf (Landgraf, 2018) has observed that a mid-waved fractal value lower than -8 [-] indicates an extremely poor ballast condition. Thus, as long as the fractal value does not exceed $-8[-]$ it is possible to improve track quality by carrying out maintenance measures - this refers to the technical possibility, but need not necessarily represent the most economic strategy. However, despite improvement due to maintenance measures the ballast condition itself remains the same. Thus, maintenance measures improve track quality and therefore the impact of poor ballast condition. The root cause of the problem (fouled ballast), however, could only be dealt with by carrying out ballast cleaning.

Figure 9 Indication mid-waved fractal analysis range.

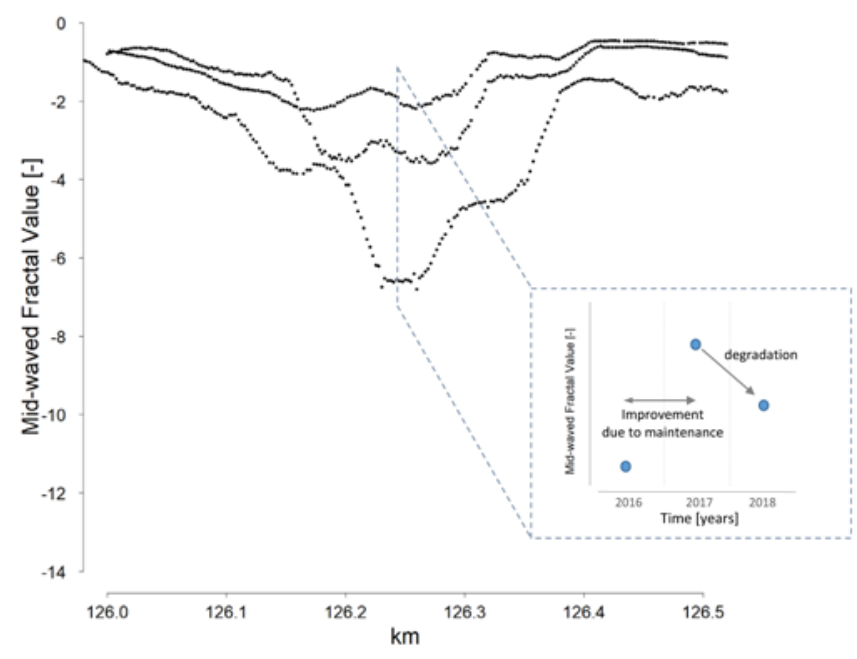

Since this was not the case, track quality improves but a high degradation rate leads to the reoccurrence of the failure in this specific area. This can be seen within Figure 9 where the high degradation between 2017 and 2018 shows the reoccurrence of the failure after a short term improvement due to maintenance between 2016 and 2017. The most current fractal values for this analysis date back to summer 2018 and the time series indicates a further degradation in ballast condition. This prognosis can be confirmed with the executed in-situ inspection (e.g. Figure 8) dated November 2018.

We can state that fractal analysis has the capability to detect and establish poor ballast condition. This leads to the question of whether DAS can achieve similar results. A precondition for using the fibre optic acoustic signal for an assessment of railway track is to link every channel to a track kilometre and furthermore to an asset. For example, when utilizing the signal for turnout condition monitoring every part of a turnout has to be linked to a corresponding channel. This means, that each insulated rail joint, the front and the rear part as well as the crossing nose of the turnout have to be linked to the channel with the highest acoustic intensity. This has the effect that any acoustic event can be linked to the root cause and it enables an asset and condition specific evaluation. The same procedure needs to be carried out for all points of interest such as track failures or other worn components.

After linking the track kilometre (e.g. Figure 8) to a channel it was possible to analyse the observed signal within the area of poor ballast condition (white spot). Once again, a prerequisite for using the raw acoustic signal of DAS is the same train type with the same train configuration. The time series of fibre acoustic sensing data dates back to October 2017 and thus it is not possible to carry out time series analysis of DAS data from 2016. However, as the first measurement with the DAS system took place in October 2017 it is possible to evaluate whether the degradation occurring between 2017 and 2018 has also 
become visible within the fibre optic acoustic signal. For this purpose, it was necessary to link the track kilometre of the white spot with the most corresponding channel of the DAS system.

Comparing the acoustic signal of the white spot (e.g. Figure 10 , left) from year 2017 with the raw acoustic signal of an open track section (e.g. Figure 6, left) - same train type, same train configuration and speed - in proper condition shows the higher acoustic intensity of the section illustrated in Figure 10 on the left-hand side. The duration and the pattern of both signals is equal which means that it is the same train type with the same configuration and speed. The acoustic signal in Figure 10 on the left-hand is generated by a train passing due to the ballast condition in 2017 on track kilometre 126.230. The ballast condition degrades between 2017 and 2018 as depicted in Figure 9. The same pattern can be observed within the raw acoustic signal. When comparing the signal from 2017 and 2018, illustrated in Figure 10, the higher acoustic intensity in 2018 is clearly visible.

Figure 10 White spot in ballast bed within DAS.

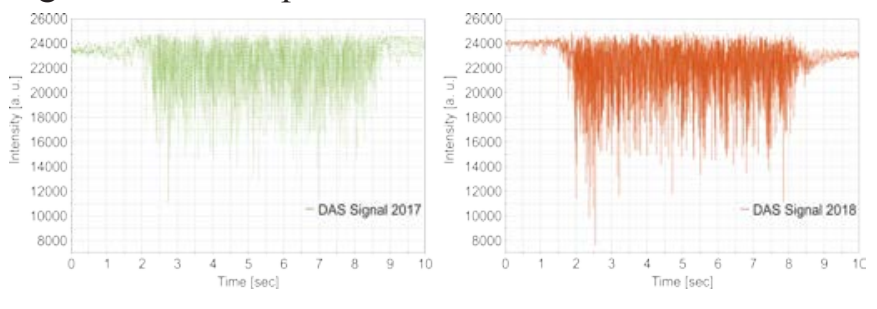

Furthermore, the acoustic signal from 2018 shows a higher intensity of the approaching train and it also stays constantly on the higher acoustic level.

It can be stated that after a thorough positioning process Distributed Acoustic Sensing has the potential to be an additive data source for infrastructure managers to evaluate the condition of a track. This evaluation potential will be especially effective in the time between the measurement runs of a track recording vehicle.

\section{Outlook/ Discussion}

The presented results give an indication of the vast area of application for Distributed Acoustic Sensing in railways. Firstly, it can be used for train tracking and safety \& security purposes. Since it is an acoustic signal this is the most obvious scope of application. But this paper goes one step further and also shows the potential of using acoustic signals for the assessment of maintenance works carried out and for railway infrastructure condition monitoring. Correlating the information from Distributed Acoustic Sensing with the evaluations based on fractal analysis of vertical track geometry shows that this innovative methodology of using fibre optic cables has the capability for assessing the condition of railway track. Fibre optic cables deliver continuous and permanent information on railway track and they also bring a very valuable addition to the results provided from the use of track recording vehicles, as this innovative approach would allow permanent behaviour monitoring between the measurement runs of track recording cars. This would be a major step forward for real-time predictive maintenance and would also lead to higher availability on railway tracks.

\section{Acknowledgement}

We would like to acknowledge our project partners Austrian Federal Railways and Swiss Federal Railways.

\section{References}

Bao, X. and Chen, L. (2012) 'Recent progress in distributed fiber optic sensors', Sensors (Basel), 12(7), pp. 8601-8639.

European Committee for Standardization (2014) EN 13848-5. Track Geometry Quality. Part 5: Geometric Quality Levels.

Frederich, F. (1984) 'Effect of track geometry on vehicle performance', ZEVrail, 108, pp. 355-362.

Hill, D. (2015) 'Distributed Acoustic Sensing (DAS): Theory and Applications', in Frontiers in Optics 2015. San Jose, California: Optical Society of America (OSA Technical Digest (online)).

Hyslip, J. (2002) 'Fractal Analysis of Track Geometry Data', Transportation Research Record: Journal of the Transportation Research Board.

Ip, E. et al. (2008) 'Coherent detection in optical fiber systems’, Optics Express. OSA, 16(2), pp. 753-791.

Landgraf, M. (2018) Smart data for sustainable Railway Asset Management. Graz: Verlag der technischen Universität Graz.

Landgraf, M. and Hansmann, F. (2018) 'Fractal analysis as an innovative approach for evaluating the condition of railway tracks', Proceedings of the Institution of Mechanical Engineers, Part F: Journal of Rail and Rapid Transit.

Lauer, M. and Stein, D. (2015) 'A Train Localization Algorithm for Train Protection Systems of the Future', IEEE Transactions on Intelligent Transportation Systems, 16(2), pp. 970-979.

Lienhart, W. et al. (2016) 'Condition monitoring of railway tracks and vehicles using fibre optic sensing techniques', in Proceedings of the International Conference on Smart Infrastructure and Construction. ICE Publishing (Cambridge Centre for Smart Infrastructure \& Construction), pp. 45-50.

Mandelbrot, B. (1967) 'How long is the coast of Britain? Statistical self-similarity and fractional dimension', Science.

Mandelbrot, B. B. and Blumen, A. (1989) 'Fractal Geometry: What is it, and What Does it do? [and Discussion]',

Proceedings of the Royal Society A: Mathematical, Physical and Engineering Sciences.

Marschnig, S. et al. (2006) 'Fahrwegstrategien für Gleis und Weiche auf komponentenbasis - Optimierung auf Basis von Lebenszykluskosten', ZEV Rail Glasers Annalen.

Owen, A. et al. (2012) 'OptaSense: Fibre optic distributed acoustic sensing for border monitoring', in Proceedings 2012 European Intelligence and Security Informatics Conference, EISIC 2012.

Papp, A. et al. (2016) 'A real-Time algorithm for train position monitoring using optical time-domain 
reflectometry', in 2016 IEEE International Conference on Intelligent Rail Transportation, ICIRT 2016, pp. 89-93.

Pohl, P. and Schubert, M. (2017) 'Fiber Optic Sensing im Eisenbahnsektor', Eisenbahningenieur, (11), pp. 41-45.

Rosenberger, M. (Frauscher S. T. (2018) 'How Distributed Acoustic Sensing supports condition based maintenance strategies', ETR International Edition, (2), pp. 28-31.

Schubert, M. and Bauer, E. (2015) 'Fiber Optic Sensing im Eisenbahnsektor', Signal+Draht, 107(9), pp. 42-46.

Timofeev, A. V et al. (2015) 'The rail traffic management with usage of C-OTDR monitoring systems', International Journal of Computer, Electrical, Automation, Control and Information Engineering, 9(7), pp. 1492-1495.

Vale, S. and Rauscher, A. (2013) 'Fiber optic based pipeline monitoring', in 8th Pipeline Technology Conference 2013. EITEP Institute.

Veit, P. (2006) 'Qualität im Gleis - Luxus oder Notwendigkeit?', Eisenbahningenieur, 57(12), pp. 32-37.

Wiesmeyr, C. et al. (2018) 'Train tracking and train condition monitoring by Distributed Acoustic Sensing', in Proceedings of 7th Transport Research Arena TRA 2018,. Vienna. 